\title{
Moustapha Safouan (1921-2020)
}

\author{
Nous publions un hommage signé de Christian Hoffmann \\ suivi de la publication d'un inédit de Moustapha Safouan \\ commenté par Christian Hoffmann ${ }^{11}$
}

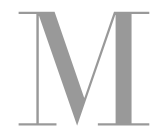

oustapha Safouan est né en 1921 à Alexandrie, il nous a quitté dans la nuit du 8 novembre 2020 pour rejoindre, comme il aimait le dire, l'“ immensité ». Centenaire, et ce signifiant avait toute son importance pour lui qui disait souvent que St Jean est mort âgé de 103 ou 104 ans.

Durant ses études universitaires de philosophie, il rencontre Émile Brehier, André Lalande, Alexandre Koyré, Jean Grenier, John Wisdom, et c'est avec Moustapha Ziwar, philosophe, médecin et psychologue, premier psychanalyste égyptien, qu'il decouvre l'œuvre de Sigmund Freud, et Ziwar lui conseillera également de faire une psychanalyse, ce qu'il a fait avec Marc Schlumberger, qui ne manquait pas de sensibilité à la langue.

Pour choisir un analyste de contrôle, il avait, comme ses camarades, le choix entre une analyse des conduites avec Daniel Lagache ou une analyse de la parole et du langage avec Jacques Lacan. Le choix de Lacan s'imposa et il devint psychanalyste en 1949. L'un des premiers à participer à son enseignement, Moustapha Safouan a suivi Lacan jusqu'a la fin de son École ; il était de tous les jurys de la Passe de l'École Freudienne de Paris.

Quand, étudiant, il a quitté une première fois l'Égypte, il voulait rejoindre Cambridge pour rencontrer Wittgenstein. Ce projet ne pouvant se réaliser pour des raisons d'administration, il vint étudier à la Sorbonne où il présente un projet de thèse à Bachelard, qu'il jettera à la poubelle à la suite de cette entrevue. Sa chance fut alors, ainsi qu'il le disait, de rencontrer Marc Schlumberger et il a fréquenté la Société psychanalytique de Paris, en 1947. Lacan l'a tout de suite intéressé, “ car il était le seul qui parlait de langage ; il parlait de la parole, ce qui fait que j’ai commencé à penser qu'il y avait quelque chose a faire en France. Dans le monde anglo-saxon, on parlait du langage mais pas de la parole. Je lui ai demandé un contrôle ${ }^{[2]}$. Il retourne en Égypte en décembre 1953 au moment où Lacan avait fait son premier séminaire a Sainte-Anne. Le coup d'État de Nasser le cloue à l'Université pendant cinq ans, tout autre travail étant impossible et il n'y avait plus de visa de sortie. C'est

[1] N.D.R.

[2] «Le grand entretien avec Moustapha Safouan », de Michel Plon, En attendant Nadeau, 9 mai 2017. 
pendant cette période en Égypte qu'il a traduit L'interpretation des reves de Freud en langue arabe, en compagnie de Greimas.

De retour à Paris, le voilà enchanté par le Séminaire de Lacan l'Éthique de la psychanalyse, il en fait un résumé, devenu célèbresans jamais être publie. Nous avions le projet de le publier avec une longue Préface. Ce travail sera publié prochainement. Réputé notamment par son art de l'interprétation dans sa pratique de la cure, il avait saisi très vite que le sujet de l'énonciation est identifié à l'objet a de Lacan, il devient aussi un travailleur infatigable, il disait souvent qu'il vivait pour travailler et qu'il ne travaillait pas pour vivre. Il considérait son travail était achevé a 98 ans avec la publication de son ouvrage chez Hermann, La civilisation post-oedipienne ${ }^{[3]}$. Il pensait mourir ce jour-là, et il est tombé malade. Il a gardé son air malicieux et son humour jusqu'à son dernier souffle, on l'entendait dire que chaque fois qu'il avançait vers la tombe, celle-ci reculait, ou qu'il allait finir par penser que la mort l'a oublié.

De sa pratique d'analyste, Il disait souvent qu'il est entre avec l'Edipe dans la psychanalyse et qu'il sort de la psychanalyse avec l'Edipe. En 50 ans, il a fait la révision de l'Edipe ${ }^{[4]}$, en inscrivant la découverte par Freud dans les restes de la chute du patriarcat lors de la société industrielle du 19eme siècle, par la découverte par les femmes du désir narcissique des hommes pour le pouvoir, ce que le théâtre de ce siècle met en scène a travers les pièces d'Ibsen et d'autres, sans oublier la philosophie de Nietzsche que Moustapha Safouan a passé des mois à lire avec beaucoup d'intérêt et de plaisir, pour arriver à soutenir dans son livre de 2015 Regard sur la civilisation oedipienne: “ l'Edipe n'est qu'une forme culturelle parmi d'autres, qui sont également possibles pour qu'elles accomplissent la même fonction, qui est la promotion de la fonction de la castration dans le psychisme ${ }^{[5]}$.

Dans son livre daté de 2013 La Psychanalyse. Science, Therapie-Et Cause ${ }^{[6]}$ il souligna que la structure dualiste mène à la mort, et que pour que la coexistence soit possible, il faut qu'il y ait une loi. Ce qui fait que le tiers, c'est d'abord la loi, la raison fraternelle, celle qui s'indique dans l'adage hégélien : il n'y a pas de satisfaction de l'un, sans la satisfaction de l'autre. Cette thèse avait déjà fait l'objet d'un livre, revu en 2010 : La parole ou la mort. Essais sur la division du sujet ${ }^{[7]}$. Il disait que ce livre est le plus lacanien qu'il ait écrit.

C'est à l'étude de cette loi, comme limite a la jouissance, et qui « nous refuse (selon Freud) la pleine satisfaction (sexuelle) » ou qui entraîne (selon Lacan) qu'il n'y a pas de rapport sexuel, que Moustapha Safouan a consacre une partie de son oeuvre depuis ses Etudes sur l'Edipe jusque dans ses ouvrages récents pour développer sa théorie du désir pris entre le sujet désirable et le sujet désirant. Après La civilisation

[3] Safouan, M., La civilisation post-œedipienne, Hermann, 2018.

[4] Safouan, M., Études sur l'CEdipe, Seuil, 1974. Reedition chez Hermann, 2018.

[5] Safouan, M., Regard sur la civilisation œdipienne Desir et finitude, Hermann, 2015, p. 179.

[6] La Psychanalyse. Science, Therapie-Et Cause, Thierry Marchaisse, 2013.

[7] La parole ou la mort, Seuil, 1993, 2010. 


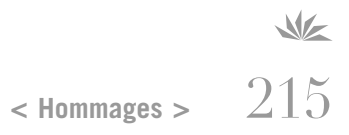

post-oedipienne, paru en 2018, nous avons encore ensemble fait un petit opuscule en 2019 sur : De la dualité à la division du sujet ${ }^{[8]}$.

Dans le livre de 2018, qu'il considérait comme l'aboutissement de son travail, il construit une analyse des mutations familiales et sociales qui ont mis la société en faillite devant l'individu néoliberal, pour qui le monde n'est que demande et satisfactions individuelles. Cette politique n'est pas sans répercussions sur l'analyse, et ceci depuis les années 70, par le changement des demandes et la transformation des grandes structures subjectives au profit de ce qu'il a nommé les “ cas limites " dans l'analyse ${ }^{[9]}$. Ces cas présentent un risque de faire un épisode psychotique pendant la cure, et, sans qu'il y ait une forclusion du signifiant du Nom-du-Père, la métaphore paternelle reste inopérante. Ceci se produit, selon lui, dans des familles marquées par l'absence de tout désir.

Moustapha Safouan est parti avec la conscience de laisser à notre charge ce programme.

Il avait indubitablement l' "étincelle " comme on le disait déjà de lui dès ses débuts. Ses Euvres completes seront publiées avec de nouvelles Préfaces et Présentations de sa plume, aux éditions Hermann.

Christian Hoffmann

[8] Safouan M., Hoffmann C., De la dualité à la division du sujet, Hermann, 2019.

[9] II a commence a parler des « cas limites » dans l'analyse dans Le grand entretien avec M. Plon, en 2017. 